\title{
Super-resolution fluorescence polarization microscopy
}

\author{
Karl Zhanghao, ${ }^{* \dagger}$, Juntao Gao ${ }^{\star \S}$, Dayong Jin", Xuedian Zhang, ${ }^{*,, \|, \dagger \dagger}$ \\ and Peng $\mathrm{Xi}^{*, \dagger, \oplus, * *, \dagger \dagger}$ \\ *Department of Biomedical Engineering, College of Engineering \\ Peking University, P. R. China \\ ${ }^{\dagger}$ School of Optical-Electronic and Computer Engineering \\ Shanghai University of Science and Technology, P. R. China \\ ${ }^{\ddagger}$ Department of Automation, Tsinghua University \\ Beijing 100084, P. R. China \\ $\S_{\text {Bioinfomatics Division, TNLIST }}$ \\ MOE Key Laboratory of Bioinformatics and \\ Center for Synthetic 85 System Biology \\ Tsinghua University, Beijing 100084, P. R. China \\ "Faculty of Science, \\ Institute for Biomedical Materials and \\ Devices (IBMD) University of Technology, Australia \\ "zhangxuedian@hotmail.com \\ **xipeng@pku.edu.cn
}

Received 28 March 2017

Accepted 8 May 2017

Published 13 June 2017

\begin{abstract}
Fluorescence polarization is related to the dipole orientation of chromophores, making fluorescence polarization microscopy possible to reveal structures and functions of tagged cellular organelles and biological macromolecules. Several recent super resolution techniques have been applied to fluorescence polarization microscopy, achieving dipole measurement at nanoscale. In this review, we summarize both diffraction limited and super resolution fluorescence polarization microscopy techniques, as well as their applications in biological imaging.
\end{abstract}

Keywords: Fluorescence polarization microscopy; super resolution; fluorescence anisotropy; linear dichroism; polarization modulation.

†Corresponding authors.

This is an Open Access article published by World Scientific Publishing Company. It is distributed under the terms of the Creative Commons Attribution 4.0 (CC-BY) License. Further distribution of this work is permitted, provided the original work is properly cited. 


\section{Introduction}

As a fundamental physical dimension of fluorescence, polarization has been applied extensively in biological researches. Through fluorescence polarization microscopy (FPM), the dipole orientation as well as the intensity of fluorescent probes could be measured. Since the orientation of the fluorescent molecule is related to the tagged biostructures, structural information of cellular organelles or macromolecules can be revealed. Although X-ray crystallography or electron microscopy could elucidate ultra-high resolution of individual proteins or macromolecule assemblies, they require very complex sample preparation unsuitable for live cell imaging. Near-field imaging techniques, such as Atomic Force Microscopy (AFM) could also provide with structural information, which however, is limited only to samples on the surface. FPM is capable of imaging orientations in dynamic samples at the time scale of seconds or milliseconds, thus it can serve as a complementary method for measurement of subcellular organelle structures. Recent decades have seen a variety of application of FPM in the area of cell membrane, biological filaments including cytoskeleton and DNA filaments, and other macromolecule assemblies.

The researches of the structure and dynamics of macromolecule assemblies demonstrate FPM as a complementary method with X-ray crystallography or electron microscopy. As an important type of biological macromolecule facilitating selective transport between nucleus and cytoplasm, the structure of nuclear pore complex (NPC) are not completely understood. With FPM, the Y-shaped nuclear pore complex subcomplexes were studied and their relative orientation according to the nuclear envelope plane could be inferred from imaging results. ${ }^{1}$ F0F1-ATPase is a kind of enzyme relating to ATP synthesis. It consists of two domains, of which the F1 motor was found to display $120^{\circ}$ stepping by FPM. ${ }^{2-4}$

Lipid membrane is generally locally uniform and provides a quasi-static environment for the fluorescent probes, the orientation of which could be easily measured. ${ }^{5-7}$ With polarized fluorescence recovery after photobleaching (FRAP), rotational diffusion of the probes could be revealed. ${ }^{8,9}$ Molecular orientation disorder in cell membrane has been found due to cholesterol depletion ${ }^{10}$ or cytoskeleton perturbation. ${ }^{11}$ Monitoring membrane proteins could also be used to observe dynamic protein activation during molecular processes such as calcium flow or protein interaction. ${ }^{12}$

Cytoskeleton provides mechanical support to maintain or deform the cell shape, and is involved in many cell signaling pathways during cytokinesis. It also provides a scaffold to organize the contents of cells in space and for intracellular transport with the motor proteins. Actin was firstly to be found to have an organized distribution of probed dipole orientations ${ }^{13}$ and was imaged by various FPM techniques. ${ }^{14-18}$ Myosin is an important motor protein moving along actin. It exhibits interesting walking pattern with steps and rotation and is an extensively studied system. ${ }^{19-24}$ Kinesin is another type of motor protein which is bound to microtubules. A highly mobile state and a rigid state of kinesin were distinguished which may relate to ATP and ADP binding. ${ }^{25}$ Other types of cytoskeleton are also studied using FPM, including microtubule ${ }^{17}$ and septin. ${ }^{18,26-29}$ Biological filamentous structures measured by FPM includes also in vitro DNA filaments $^{17,30}$ and human amyloid fibrils. ${ }^{31}$

FPM has been evolving during past decades, from manual or mechanical switching of polarization detection or excitation to simultaneous detection and fast polarization modulation via electro-optic devices. With faster imaging speed and higher imaging quality, FPM has been incorporated with various imaging modalities, such as wide-field, ${ }^{28,29}$ confocal, ${ }^{11,32}$ two-photon confocal, ${ }^{10,12,33-35}$ total internal reflection fluorescence, ${ }^{19}$ FRAP,${ }^{8,9}$ etc. However, as an optical imaging technique, the development of fluorescence polarization microscopy (FPM) is barricaded by the diffraction limit. Compared to the abundant super-resolution techniques on fluorescence intensity imaging, super-resolution techniques in FPM is still in its infancy. In the review, we would firstly summarize current FPM techniques and then introduce recently developed two kinds of superresolution techniques in FPM. We would conclude the review with a comparison of the advantages and disadvantages between various FPM techniques and outlook its application in the future.

\section{Principle of Fluorescence Polarization Microscopy}

Optical properties of most chromophores are anisotropic, with transition moments along a specific 
direction in the molecular structure. Fluorescent dipoles could be used to model excitation absorption or fluorescence emission of chromophores, which remain the same in most cases. ${ }^{36}$ When excited by polarized light, the dipoles with their orientations aligned parallel to the electric vector of the polarized excitation would have the highest probability of absorption. The distribution of absorption probability on polarized excitation is proportional to $\cos ^{2} \theta$, where $\theta$ is the intersecting angle between absorption dipole orientation and polarization direction. Discussion of two-photon or multi-photon absorption is more complex and not included here. ${ }^{37,38}$ The emitted fluorescence from dipoles is also polarized, with the highest intensity component of polarization along the emission dipole orientation. The distribution of intensity probability on emission polarization is proportional to $\cos ^{2} \theta^{\prime}$, where $\theta^{\prime}$ is the intersecting angle between emission dipole orientation and the polarization direction of the polarized component of emitted fluorescence. According to the anisotropy in fluorescent absorption or emission, the dipole orientations of chromophores could be measured. In real conditions, the linker between fluorescent probes and the structure attached is not absolutely firm, which makes the dipoles wobbling at a speed much faster than the imaging speed. Thus, the real fluorescent dipoles are modeled with average azimuth and wobbling angle in many researches. ${ }^{12,17,33}$

Fluorescence Anisotropy (FA) utilizes the polarization of emitted fluorescence. A typical FA setup consists of linearly polarized excitation and two polarized detections of fluorescence, which are parallel or perpendicular to the direction of the polarized excitation. The parallel and perpendicular detection could be achieved sequentially by changing analyzers in the emission path or simultaneously by polarization beam splitter (PBS), ${ }^{13}$ a Wollaston prism ${ }^{17}$ or a Thompson prism. ${ }^{39}$ The anisotropy and azimuth could be calculated through two relating pixels. Early FA research started from studying the fluorescence polarization of chromophores in solutions ${ }^{40}{ }^{4}$ which did not include an objective to collimate the fluorescence. An objective would greatly improve collected signals but may influence the fluorescence polarization when numeric aperture (N.A.) is high. A general theory for epi-illumination observation of FA through high N.A. objectives was presented by Axelrod ${ }^{5}$ and was applied to measure diI - a kind of membrane probes - on erythrocyte ghosts. FA was later implemented with total internal reflection fluorescence microscopy (TIRFM) ${ }^{19}$ fluorescence recovery after photobleaching (FRAP), ${ }^{8}$ etc. Four detection channels could enable 3D orientation detection of fluorescent dipoles instead of only in-plane measurement, ${ }^{41}$ or could provide an unbiased measurement of dipole orientations. $^{16}$

Based on emitted fluorescence polarization of radiating dipoles, the fluorescent intensity distribution outside the objective image space could also provide orientational information. ${ }^{42,43}$ When the dipole lies at an orientation unparalleled to the optical axis, the polarized emission would lead to rotationally asymmetric distribution in the annular image. The orientation could be retrieved from the asymmetric image via pattern analysis or pattern recognition. The orientational information within the defocused pattern is not only limited to in-plane angle but also out-of-focus tilting angle ${ }^{44}$ Besides, the dipole orientation is found to strongly relate with the molecular localization position. Later, a similar principle was applied to wide-field epifluorescence microscopy and dipole orientations could be measured from the characteristic intensity distribution of the defocused image. ${ }^{45} \mathrm{~A}$ pattern recognition algorithm based on least-squares analysis was developed to fit the calculated set of master patterns against measured images, which could be applied to determine three-dimensional orientations in defocused single-dipole images. ${ }^{46}$ Because of its principle, this technique is termed defocused pattern recognition (DPR) in the review. Based on this principle, Defocused Orientation and Position Imaging (DOPI) measured 3D orientation and stepping behavior of the light-chain domain of myosin $\mathrm{V}$ as myosin $\mathrm{V}$ moves along actin. ${ }^{23}$

While both FA and DPR determine dipole orientations via emitted fluorescence polarization, linear dichroism (LD) is based on the absorption anisotropy of chromophores. LD exploits rotary linear polarized excitation and records the sinusoidal response of the fluorescence. The orientation of fluorescent dipoles could be extracted from the polarization modulation data by curve fitting or Fourier analysis. Rotary linear polarized excitation could be achieved by passing a circularly polarized light through a rotary polarizer ${ }^{2}$ or by passing a linear polarized light through a rotary half wave plate. ${ }^{12,15,18}$ Rotary devices are limited in modulation speed and face with averaging effects due to 


\section{K. Zhanghao et al.}

their continuous motion. Electro optic devices could provide fast and discrete modulation of lasers, including the Pockel cell, ${ }^{19}$ liquid crystal variable retarder, ${ }^{6,28}$ electro-optic modulator. ${ }^{25,30} \mathrm{LD}$ is easy to combine with epi-illumination wide field microscopy and laser scanning confocal microscopy, with additional polarization modulating devices. The polarization of the focal point of confocal illumination is linearly polarized in-plane if the excitation is polarized. ${ }^{12}$ Experiments also demonstrated the markedly higher sensitivity of twophoton LD than single photon microscopy. ${ }^{12,33}$ To further improve imaging speed together with optical sectioning, LD was also incorporated with a spinning disk confocal microscope. ${ }^{32}$ Unlike FA, LD could easily exceed two orthogonal polarized excitations. $^{7,29}$ Four polarizations $\left(0^{\circ}, 45^{\circ}, 90^{\circ}\right.$, $\left.135^{\circ}\right)^{19,28}$ or more ${ }^{15}$ are widely used. Some researchers hold the view that four polarizations are enough for sampling the sinusoidal curve and for an unbiased measurement of the in-plane dipole orientation. ${ }^{16,28}$ Eight combinations of polarization could allow unambiguous 3D measurement of dipole orientations. ${ }^{47}$

Most FPM could only measure in-plane dipole orientations while some could access out-of-plane tilting angle of dipoles as well. DPR could extract $3 \mathrm{D}$ orientation of single dipole when $z$ position information of the dipole is combined..$^{23,44,45} 3 \mathrm{D}$ orientation measurement with FA was also proposed. ${ }^{41,48}$ LD with epi-illumination could not provide axially polarized excitation, which however, exists in highly inclined thin illumination (HILO) ${ }^{49}$ or in the illumination of total internal reflection microscopy. Such design could be achieved by a rotary combination of a half wave plate and a prism, ${ }^{3}$ or polarization modulation with a Pockel cell, as in polTIRF. ${ }^{19,47,50}$ LD has also been combined with multi-focus imaging which could achieve $2 \mathrm{D}$ orientation measurement and $3 \mathrm{D}$ intensity imaging. ${ }^{26}$

Polarization Distortion is a general issue in FPM, which requires special attention. Optical elements like lenses, reflection mirrors, etc. would not bring any distortion, while most polarization distortion is from the dichroic mirror. Other devices like the spinning disk unit, fibers are alternative sources. Polarization distortion of the dichroic mirror could be approximately compensated by a quarter wave plate $^{11}$ or be accurately handled via a Berek's polarization compensator or the Soleil Babinet compensator. ${ }^{32}$ System calibration of the polarization distortion could be measured by a polarizer placed on top of the objective when epi-illumination is used. Standard samples with known dipole orientation, such as fixed single molecules, GFP crystals, ${ }^{51}$ in vitro fluorescent labeled actin samples, ${ }^{52}$ are perfect for calibrating the system.

Both FA and LD are based on the analysis of the fluorescence polarization, either in excitation or in emission. FA setup seems to be more simple and could achieve simultaneous observation. However, the number of detection channels is usually limited by the total fluorescence signal. FA would also be affected by depolarization processes, such as resonance energy transfer, rotational diffusion, etc. Though these properties could provide FA with capabilities to image related phenomenon, they would bring errors in measuring the transition moments of dipoles. In contrast, LD is weakly affected by depolarization processes. It could have more sampling points within one modulation period, though it needs multiple frames for final results. Since FA and LD are separately placed in the detection path and in the excitation path, they are complementary other than being conflict to each other. In some cases, LD and FA are implemented in the same instruments for specific purposes. ${ }^{19,47,50}$

\section{Super-Resolution Techniques in FPM}

Ernst Abbe proved that the resolution of optical microscopes would be limited by the optical diffraction, ${ }^{53}$ which is about $250 \mathrm{~nm}$ in lateral resolution for far-field optical imaging. Super-resolution techniques break the diffraction barrier, which is achieved through intensity on-off modulation. The modulation could be in a structured manner, such as stimulated emission depletion (STED) $)^{54,55}$ or (saturated) structured illumination microscopy (SIM),${ }^{56-58}$ or in a stochastic manner, such as (f) photo-activated localization microscopy (PALM)/ stochastic optical reconstruction microscopy (STORM). ${ }^{59-62}$ Though a large variety of super resolution techniques have been developed for fluorescence intensity imaging, super resolution FPM is just starting. To achieve super resolution with dipole orientation measurement, one simple thought is to combine FPM with existing super resolution techniques, such as STED, SIM, PALM/STORM, etc. 
The other thought is to exploit the intensity modulation of LD, which modulates the excitation laser and gets a sinusoidal response of the sample. Recently, developed super resolution FPM techniques could be categorized into two group: one is based on intensity modulation of $\mathrm{LD}^{15,18}$ and the other one is to combine FA with direct stochastic optical reconstruction microscopy (dSTORM) ${ }^{17}$

In Ref. 15, Hafi et al. developed super resolution by polarization demodulation $(\mathrm{SPoD})$ and excitation polarization angle narrowing (ExPAN) which achieved super resolution with neither structured illumination nor special switchable or blinking fluorescent probes. ${ }^{15}$ They applied the technique to standard wide-field microscopy and to two-photon scanning microscopy. Two excitation laser beams with rotary polarization were used, with a deexciting stimulated emission beam of always perpendicular polarization to the excitation beam. By doing this, ExPAN could narrow the range of polarization angles that results in effective excitation of differently oriented molecules. ExPAN could determine the orientation of fluorescent markers attached to structures and measure changes more accurately. Together with SPoD, ExPAN was also demonstrated to bring a better spatial resolution of overlapping fluorescent molecules.

SPoD achieves super resolution imaging by detecting the periodic signals emitted with different phases from different nanoareas under rotary polarized excitation. The rotary polarization of both excitation and stimulated beams is done by passing them through the same rotary half wave plate. The polarization modulation data contains 10 sampling point during each cycle and is demodulated by the sparsity penalty-enhanced estimation by demodulation (SPEED) deconvolution algorithm. The algorithm models each nanoarea as a mean dipole and distinguish neighboring emitters within the diffraction limited area through the different phases of sinusoidal response to rotary polarized excitation, i.e., through different dipole orientations of the mean dipoles. To achieve this, a model of the polarization modulated system is built and penalized maximum likelihood estimation is utilized to estimate original sample information. Fast iterative shrinkage-thresholding algorithm (FISTA) solves the deconvolution problem.

Though SPoD with ExPAN achieved super resolution through polarization demodulation, the information of dipole orientations is lost during the deconvolution process. Hence, SPoD could not be strictly taken as a form of FPM. There also has been an interesting debate on whether polarization modulation adds super resolution or not. ${ }^{63,64}$ Super resolution dipole orientation mapping (SDOM) extended SPoD with measurement of dipole orientations, which adds promptly evidence to the debate. $^{18}$

Instead of the SPEED algorithm in SPoD, SDOM establishes a polarization-variant model, in which the intensity determines the super-resolution microscopic image using sparsity-enhanced deconvolution, while the phase determines the effective dipole orientation of each super-resolved focal volume using least squares estimation, thus fully exploiting the polarization modulation information. The dipole orientations are superimposed onto the image as arrows, whose direction and length denotes effective dipole orientation and orientation uniformity accordingly. SDOM achieves comparable intensity resolution compared to SPoD while achieving super resolution dipole orientation mapping as well. The imaging speed of SDOM could be 5 f.p.s and requires low excitation power, making it possible for live cell imaging of yeast.

Both SPoD and SDOM utilize polarization modulation to modulate the intensity of fluorescence. However, it is limited by the small probability that all fluorophores have the same orientation in diffraction-limited zones of labeled samples. When the sample consists of high densities stochastically oriented fluorophores or is highly dynamic in orientation, it would be hard to detect fluorescence modulation.

Another super-resolution technique is based on single molecular imaging. Single molecular imaging of fluorescent dipoles in intensity and orientation has long been investigated. ${ }^{16,19,23,48}$ For LD single dipole measurement, two or four polarization excitations are modulated for the balance of dipole orientation measurement and imaging speed. ${ }^{19,47}$ Simultaneous imaging brings FA the advantage over LD of faster imaging. However, splitting the fluorescent signal into multiple detection channels would reduce the signal-to-noise ratio, thus limiting the number of detection channels. In most cases, two detection channels are used. Four channels could achieve $3 \mathrm{D}$ orientation detection ${ }^{41}$ or unambiguous measurement of the dipole's average orientation and wobbling angle. ${ }^{16}$ However, this only applies to very diluted labeling of fluorophores and 


\section{K. Zhanghao et al.}

could hardly reveal complex bio-structures. This could be solved by polarization-resolved direct stochastic optical reconstruction microscopy (polardSTORM), ${ }^{17}$ which measures single fluorescent dipoles in one frame, and switches to other dipoles on the ON state stochastically in other frames, accumulating a full picture of the samples from adequate frames.

To preserve high signal-to-noise ratio for single molecular localization, two emission detection channels were used, which allows in-plane orientation measurement only and omits the wobbling information of single dipoles. The designed algorithm estimates both the azimuth and the position of the single molecules in each frame, which provides accurate localization and orientation measurement. With single molecular detection in polar-dSTORM, the dipole information measured includes the average orientation of the dipole and the wobbling aperture angle. The average orientation of the dipole could be measured directly from the fluorescence anisotropy in each frame, while the wobbling aperture angle is statically calculated from nearby localized emitters. Previous FPM images could only offer an average of orientation information over many molecules, which would lose information on individual wobbling behaviors at some extent. It was found that the average orientation may remain the same in ordered and disordered system while their wobbling angle varies. Polar-dSTORM was applied to nanoscale orientational order imaging of biological filaments, including dsDNA, actin, and microtubule, providing quantitative results of point orientation and wobbling angle. Since the imaging time of polar-dSTORM takes 2-40 min and statistical calculation of molecules detected during the period is used, it fits better for stationary samples. The specific sample preparation of polardSTORM also limits the application of live cells.

\section{Discussion and Conclusion}

Diffraction limited FPM techniques are generally applied to imaging bulk samples with organized distribution of dipole orientations. Taking the study of septin for example, both early research and FluoPolScope could only reveal the $90^{\circ}$ rotation of the filament direction. ${ }^{27-29}$ As for the intermediate state, during which the filament direction transits, they could hardly do anything and requires higher resolution imaging. ${ }^{65}$ What's worse, the results true for the bulk sample, may be completely wrong for single fluorescent probes, ${ }^{17,18}$ making the measurement results unreliable.

Single molecular dipole imaging could achieve both position tracking and dipole orientation measurement of single molecules at microseconds time scale. The localization precision could be as high as

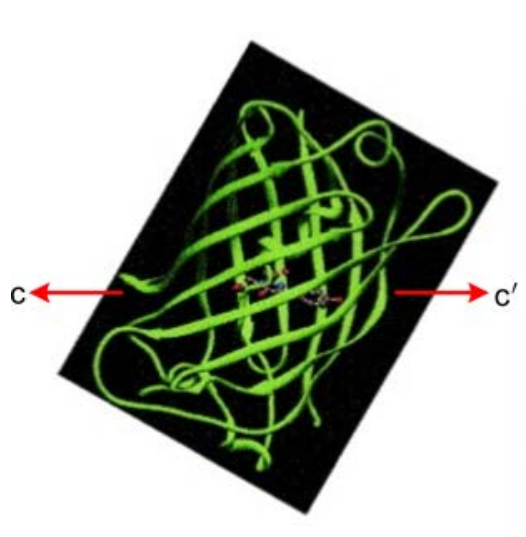

(a)
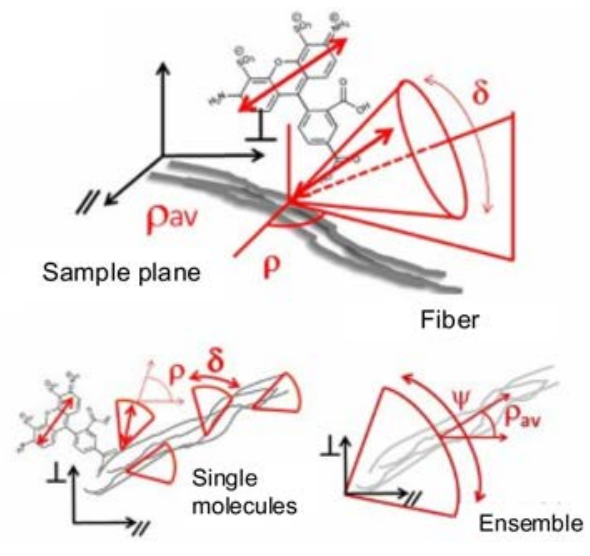

(b)

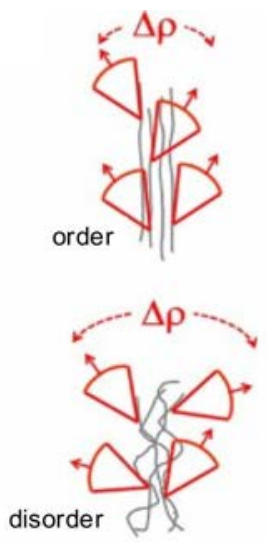

disorder 


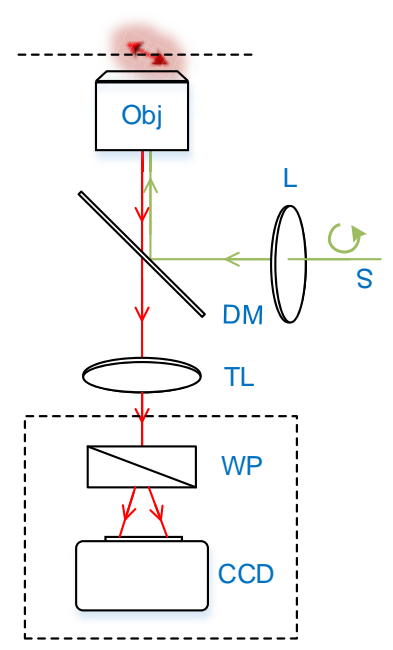

(a)

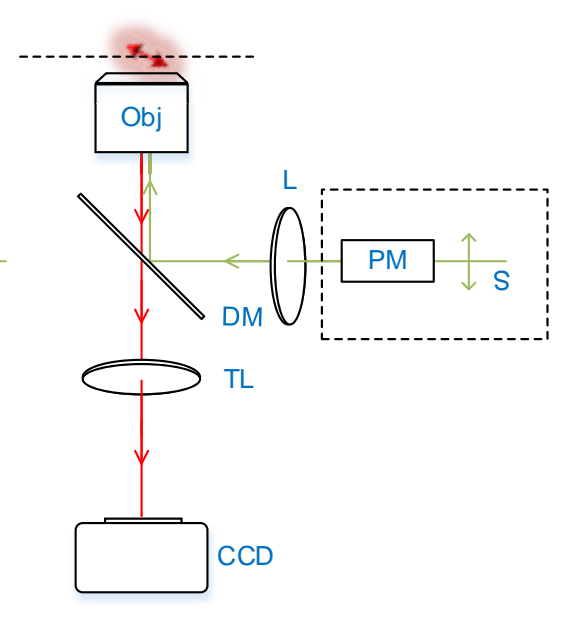

(b)

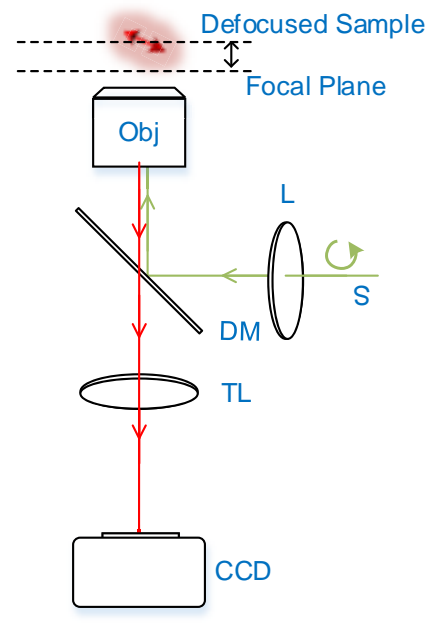

(c)

Notes: Abbreviations: S - light source; L - collimating lens; DM - dichroic mirror; Obj - objective; TL - tube lens; CCD - CCD camera; WP Wollaston Prism; PM - polarization modulator.

Fig. 2. Schematic setup of different types of FPM. (a) A typical setup of FA. Circularly polarized light source is used, with either epi-illumination (displayed in the figure) or confocal illumination (together with point detectors, not displayed). Wollaston Prism (WP) could allow simultaneous detection of two polarization channels utilizing two halves of the camera. (b) A typical setup of linear dichroism (LD). The linearly polarized light source passes a polarization modulator (PM), resulting in varying polarizations of excitation light. The time trace images of fluorescence intensity changing with the excitation polarization would be recorded. Both epi-illumination or confocal illumination could be used and the polarization modulator could be a rotary half wave plate, an electrooptical modulator, etc. (c) A typical setup of defocused pattern recognition (DPR). The sample is usually defocused with several hundred nanometers away from the focal plane of the objective. The diffraction pattern of the dipoles would be recorded and be used for orientation measurement. Circularly polarized light source is used for effective excitation of all the dipoles.

several nanometers. It achieved great success in studying the rotational walking of motor protein myosin $^{19,23,47,50}$ and the step rotation of ATPase enzyme. ${ }^{2,3,65}$ Whereas, single dipole imaging is limited particle tracking or samples with dilute labeling. It lacks the power to reveal samples with complex structure. Polar-dSTORM solves this by on-off modulation of the fluorescent probes and acquisition adequate frames for a reconstruction of super resolution image. The imaging resolution of polar-dSTORM is high, with localization precision of tens of nanometers. Single dipole average orientation is directly measured separately and the wobbling angle is statistically calculated from neighboring emitters. The drawback of polardSTORM is long imaging time of $2-40 \mathrm{~min}$, which requires a stationary sample during the imaging period. The sample preparation of dSTORM also makes it hard for live cell samples.

SDOM has achieved super resolution dipole orientation mapping with a spatial resolution of $150 \mathrm{~nm}$ and sub-second temporal resolution. It has been applied to both fixed cell and live cell imaging, which shows great advantages over diffraction limited FPM techniques on both revealing subdiffractional structures and measuring local dipole orientations. In comparison with polardSTROM, SDOM still measures average dipoles and could not separate the signal of the wobbling of single fluorophores from the variation of orientation distribution of fluorophores with the resolvable area. Same with SPoD, the power of SDOM would be weakened if the fluorescent probes are distributed too homogeneously or too dense. A comparison of actin imaging results among SDOM, polardSTORM and instantaneous FluoPolScope are displayed in Fig. 4. Summary of more representative FPM techniques is included in Table 1, together with related literatures and their characteristics.

Thanks to the intrinsic polarization of chromophores, fluorescence polarization reveals the structures and functions of the biological macromolecules. With incorporation with various optical imaging modalities, FPM has played an irreplaceable role in 


\section{K. Zhanghao et al.}
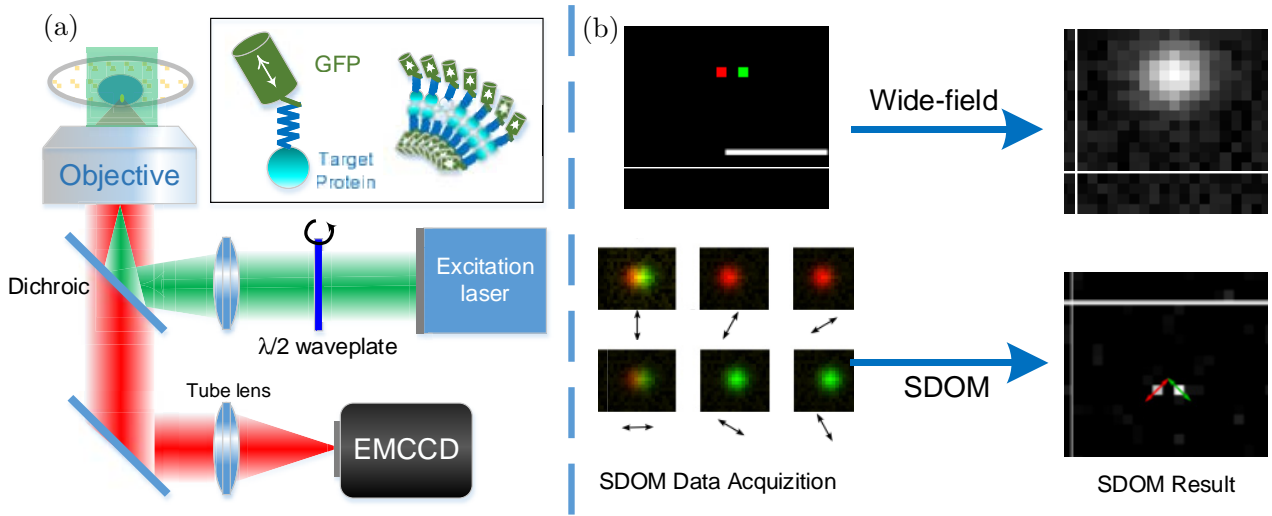

SDOM Data Acquizition

SDOM Result

(c)

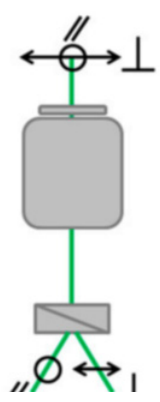

(d) 1

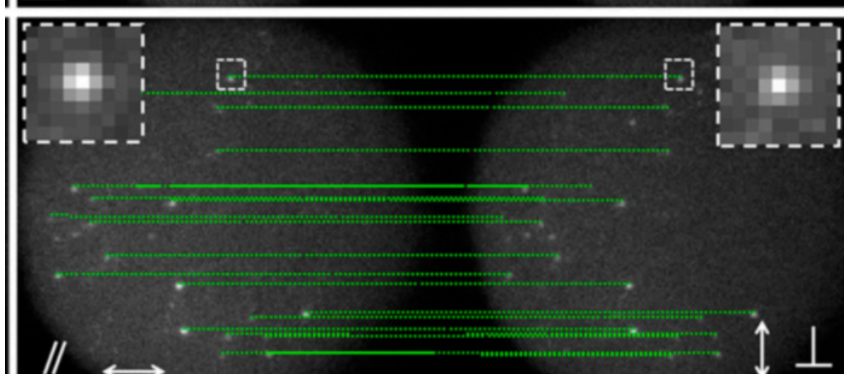

Fig. 3. Principle of SDOM and polar-dSTORM. (a) Setup of SDOM: The rotary linearly polarized laser is realized by continuously rotating a half-wave plate and incorporated into a wide-field epi-fluorescence illumination microscope. The polarization-modulated images are collected by an EMCCD camera, which is in synchronization with the rotary mount. When the fluorophores are linked to the target protein, the dipole orientations indicate the structural information of target proteins. (b) Illustration of the principle of SDOM: through linearly polarized excitation, two neighboring fluorophores (pseudo color in red and green) with $100 \mathrm{~nm}$ distance and different dipole orientations emit periodic signals. With SDOM, two emitters can be distinguished and the dipole orientation can be determined. Arrows indicate the directions of dipole orientations. (c) Schematic setup of polar-dSTORM: circularly polarized laser is used for epi-illumination. The perpendicular $(\perp$, the vertical axis of the sample plane) and parallel ( $\|$, the horizontal axis of the sample plane) polarization states are split by a Wollaston prism and recorded by the EMCCD camera. (d) Principle of polardSTORM: two recorded polarization channels of one frame are simultaneously recorded and analyzed. Single dipoles are localized and measured with in-plane orientations. Figures adapted from Refs. 17 and 18.

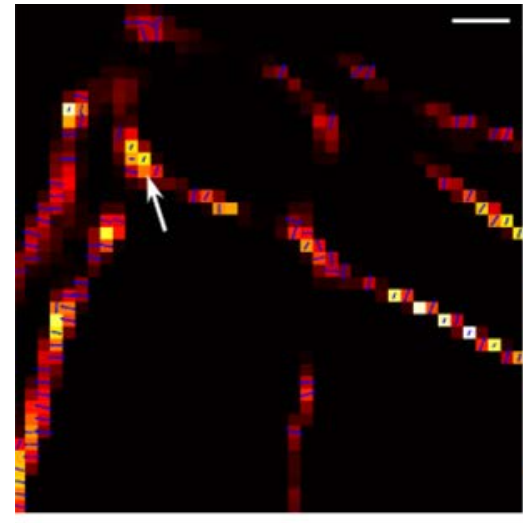

(a)

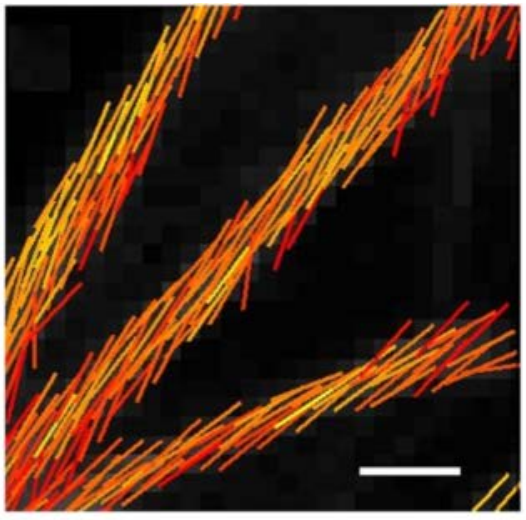

(b)

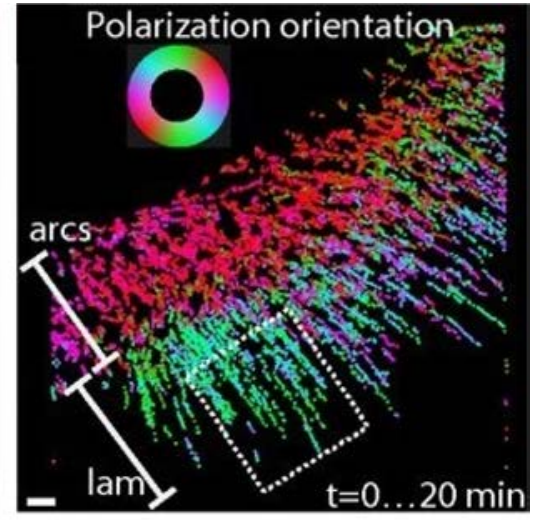

(c)

Fig. 4. Super resolution dipole measurement on actin samples; (a) SDOM results of actin in fixed cells. The dipole orientation is marked with arrows on each pixel. (b) Polar-dSTORM results of actin in fixed cells. The direction of line segments indicates the orientation of single dipoles. (c) Temporal projection of tracking results of actin in live cells by instantaneous FluoPolScope. The dipole orientation is coded with pseudo color in the color wheel. The bands corresponding to actin arcs (arcs) and the bands corresponding to lamellipodium (lam) behave different polarization orientations (b) is adapted from Ref. 17 and (c) is adapted from Ref. 16. Scale bar: a, $200 \mathrm{~nm}$; b, $1.5 \mu \mathrm{m} ; \mathrm{c}, 1 \mu \mathrm{m}$. 
Table 1. Comparison between selective fluorescence polarization microscopy techniques.

\begin{tabular}{|c|c|c|c|c|}
\hline SR-FPM techniques & $\begin{array}{l}\text { Representative } \\
\text { literatures }\end{array}$ & Basic principle & Advantages & Limitations \\
\hline FluoPolScope & Refs. 26 and 28 & LD with wide field & $\begin{array}{l}\text { Relative fast speed ( } 0.2 \text { f.p.s); } \\
\text { 3D imaging with multi-focus } \\
\text { imaging }\end{array}$ & $\begin{array}{l}\text { Diffraction-limited } \\
\quad \text { imaging; } \\
\text { Ensemble orientation; }\end{array}$ \\
\hline polTIRF & Refs. 19 and 47 & $\begin{array}{l}\text { LD and FA with } \\
\text { Total Internal } \\
\text { Reflection } \\
\text { Fluorescence } \\
\text { Microscopy }\end{array}$ & $\begin{array}{l}\text { Combination of LD and FA; } \\
\text { Fast imaging speed ( } 25 \text { f.p.s); } \\
\text { Tilting angle measurement }\end{array}$ & $\begin{array}{l}\text { Limited to dilute } \\
\text { labeling (or } \\
\text { diffraction-limited } \\
\text { imaging) }\end{array}$ \\
\hline $2 \mathrm{PPM}$ & Refs. 10 and 12 & $\begin{array}{l}\text { LD with two-photon } \\
\text { confocal }\end{array}$ & $\begin{array}{l}\text { Narrow polarization excitation } \\
\text { via two-photon absorption; } \\
\text { Optical sectioning of confocal }\end{array}$ & $\begin{array}{l}\text { Diffraction-limited } \\
\quad \text { imaging; } \\
\text { Ensemble orientation; }\end{array}$ \\
\hline DOPI & Refs. 23 & DPR & $\begin{array}{l}\text { 3D orientation measurement; } \\
\text { Fast tracking ( } 1.5 \text { f.p.s); }\end{array}$ & $\begin{array}{l}\text { Limited to dilute } \\
\text { labeling }\end{array}$ \\
\hline $\begin{array}{l}\text { Instantons } \\
\text { FluoPolScope }\end{array}$ & Ref. 16 & $\mathrm{FA}$ & $\begin{array}{l}\text { Unambiguous measurement } \\
\text { of in-plane orientation; } \\
\text { Fast tracking (10 f.p.s); }\end{array}$ & $\begin{array}{l}\text { Limited to dilute } \\
\text { labeling (or } \\
\text { diffraction-limited } \\
\text { imaging) }\end{array}$ \\
\hline Polar-STORM & Ref. 17 & FA; SR via dSTORM & $\begin{array}{l}\text { Super resolution imaging with } \\
\text { localization precision of } \\
30 \mathrm{~nm} \text {; } \\
\text { Measurement of single dipole } \\
\text { orientation and wobbling; }\end{array}$ & $\begin{array}{l}\text { Long imaging time } \\
\quad(2-40 \mathrm{~min})\end{array}$ \\
\hline SPoD with ExPAN & Ref. 15 & $\begin{array}{l}\mathrm{LD} ; \mathrm{SR} \text { via } \\
\text { polarization } \\
\text { demodulation }\end{array}$ & $\begin{array}{l}\text { Narrow polarization excitation } \\
\text { via stimulated depletion; } \\
\text { Fast SR imaging ( } 3 \text { f.p.s); }\end{array}$ & $\begin{array}{l}\text { Unable to measure } \\
\text { dipole orientation }\end{array}$ \\
\hline SDOM & Ref. 18 & $\begin{array}{l}\text { LD; SR via } \\
\text { polarization } \\
\text { demodulation }\end{array}$ & $\begin{array}{l}\text { Super resolution measurement } \\
\text { of intensity and dipole } \\
\text { orientation; } \\
\text { Fast SR imaging ( } 5 \text { f.p.s.); }\end{array}$ & Ensemble orientation \\
\hline
\end{tabular}

solving many questions. Fast and noninvasive imaging of the samples makes it a complementary tool for $\mathrm{X}$-crystallography which typically applies to individual proteins, or sub-complexes, or EM which requires invasive sample preparation, or AFM which could measure the surface of the sample. Compared to these methods, the specific labeling of the fluorescent probes provides better focus on the structure of interest. As the development of FPM techniques, its power has spread from uniform oriented fluorophores to fluorescent dipoles with organized orientation or on complex bio-structures. The detection accuracy has improved from measuring the bulk volume polarization to sub-diffraction area measurement and single dipole measurement. Imaging resolution of FPM matters not only for intensity image but also for the accuracy of dipole orientation detection. Recently developed super resolution FPM techniques still have their limitations though demonstrating great successes in their imaging results. Spatial 3D super resolution FPM techniques and 3D orientation measurement of fluorescent dipoles are still missing. In the future, we anticipate more inventions which could achieve both high-resolution measurement and fast temporal resolution, allowing imaging samples of live cells. This may be done by introducing existing super resolution principles into FPM, or by better exploiting the intensity fluctuation with polarization modulation, or other alternative means.

\section{Acknowledgments}

This work is supported by the National Instrument Development Special Program (2013YQ03065102), the Natural Science Foundation of China (614-75010, 
61428501), and Science and Technology Commission of Shanghai Municipality (16DZ-1100300).

\section{References}

1. M. Kampmann, C. E. Atkinson, A. L. Mattheyses, S. M. Simon, "Mapping the orientation of nuclear pore proteins in living cells with polarized fluorescence microscopy," Nat. Struct. Mol. Biol. 18, 643649 (2011).

2. K. Adachi, R. Yasuda, H. Noji, H. Itoh, Y. Harada, M. Yoshida, K. Kinosita, "Stepping rotation of F-1ATPase visualized through angle-resolved singlefluorophore imaging," Proc. Natl. Acad. Sci. USA 97, 7243-7247 (2000).

3. T. Nishizaka, K. Oiwa, H. Noji, S. Kimura, E. Muneyuki, M. Yoshida, K. Kinosita Jr., "Chemomechanical coupling in F1-ATPase revealed by simultaneous observation of nucleotide kinetics and rotation," Nat. Struct. Mol. Biol. 11, 142-148 (2004).

4. D. Sabbert, S. Engelbrecht, W. Junge, "Intersubunit rotation in active F-ATPase," Nature 381, 623-625 (1996).

5. D. Axelrod, "Carbocyanine dye orientation in red cell membrane studied by microscopic fluorescence polarization," Biophys. J. 26, 557-573 (1979).

6. J. F. Lesoine, J. Y. Lee, J. R. Krogmeier, H. Kang, M. L. Clarke, R. Chang, D. L. Sackett, R. Nossal, J. Hwang, "Quantitative scheme for full-field polarization rotating fluorescence microscopy using a liquid crystal variable retarder," Rev. Sci. Instrum. 83, 053705 (2012).

7. G. J. Schutz, H. Schindler, T. Schmidt, "Imaging single-molecule dichroism," Opt. Lett. 22, 651-653 (1997).

8. D. R. Fooksman, M. Edidin, B. G. Barisas, "Measuring rotational diffusion of MHC class I on live cells by polarized FPR," Biophys. Chem. 130, 10-16 (2007).

9. M. Velez, D. Axelrod, "Polarized fluorescence photobleaching recovery for measuring rotational diffusion in solutions and membranes," Biophys. J. 53, 575-591 (1988).

10. R. K. P. Benninger, B. Onfelt, M. A. A. Neil, D. M. Davis, P. M. W. French, "Fluorescence imaging of two-photon linear dichroism: Cholesterol depletion disrupts molecular orientation in cell membranes," Biophys. J. 88, 609-622 (2005).

11. A. Kress, X. Wang, H. Ranchon, J. Savatier, H. Rigneault, P. Ferrand, S. Brasselet, "Mapping the local organization of cell membranes using excitation-polarization-resolved confocal fluorescence microscopy," Biophys. J. 105, 127-136 (2013).
12. J. Lazar, A. Bondar, S. Timr, S. J. Firestein, "Twophoton polarization microscopy reveals protein structure and function," Nat. Methods 8, 684-690 (2011).

13. K. Kinosita Jr., H. Itoh, S. Ishiwata, K. Hirano, T. Nishizaka, T. Hayakawa, "Dual-view microscopy with a single camera: Real-time imaging of molecular orientations and calcium," J. Cell Biol. 115, 67-73 (1991).

14. E. Betzig, R. J. Chichester, "Single molecules observed by near-field scanning optical microscopy (Nsom)," Biophys. J. 66, A277-A277 (1994).

15. N. Hafi, M. Grunwald, L. S. van den Heuvel, T. Aspelmeier, J.-H. Chen, M. Zagrebelsky, O. M. Schütte, C. Steinem, M. Korte, A. Munk et al., "Fluorescence nanoscopy by polarization modulation and polarization angle narrowing," Nat. Methods 11, 579-584 (2014).

16. S. B. Mehta, M. McQuilken, P. J. La Riviere, P. Occhipinti, A. Verma, R. Oldenbourg, A. S. Gladfelter, T. Tani, "Dissection of molecular assembly dynamics by tracking orientation and position of single molecules in live cells," Proc. Natl. Acad. Sci. 113, E6352-E6361 (2016).

17. C. A. Valades Cruz, H. A. Shaban, A. Kress, N. Bertaux, S. Monneret, M. Mavrakis, J. Savatier, S. Brasselet, "Quantitative nanoscale imaging of orientational order in biological filaments by polarized superresolution microscopy," Proc. Natl. Acad. Sci. 113, E820-E828 (2016).

18. K. Zhanghao, L. Chen, X.-S. Yang, M.-Y. Wang, Z.-L. Jing, H.-B. Han, M. Q. Zhang, D. Jin, J.-T. Gao, P. Xi, "Super-resolution dipole orientation mapping via polarization demodulation," Light Sci. Appl. 5, e16166 (2016).

19. J. N. Forkey, M. E. Quinlan, M. A. Shaw, J. E. Corrie, Y. E. Goldman, "Three-dimensional structural dynamics of myosin $\mathrm{V}$ by single-molecule fluorescence polarization," Nature 422, 399-404 (2003).

20. M. Irving, T. St Claire Allen, C. Sabido-David, J. S. Craik, B. Brandmeier, J. Kendrick-Jones, J. E. Corrie, D. R. Trentham, Y. E. Goldman, "Tilting of the light-chain region of myosin during step length changes and active force generation in skeletal muscle," Nature 375, 688-691 (1995).

21. C. Sabido-David, S. C. Hopkins, L. D. Saraswat, S. Lowey, Y. E. Goldman, M. Irving, "Orientation changes of fluorescent probes at five sites on the myosin regulatory light chain during contraction of single skeletal muscle fibres," J. Mol. Biol. 279, 387-402 (1998).

22. I. Sase, H. Miyata, S. Ishiwata, K. Kinosita, "Axial rotation of sliding actin filaments revealed by singlefluorophore imaging," Proc. Natl. Acad. Sci. USA 94, 5646-5650 (1997). 
23. E. Toprak, J. Enderlein, S. Syed, S. A. McKinney, R. G. Petschek, T. Ha, Y. E. Goldman, P. R. Selvin, "Defocused orientation and position imaging (DOPI) of myosin V," Proc. Natl. Acad. Sci. 103, 6495-6499 (2006).

24. D. M. Warshaw, E. Hayes, D. Gaffney, A. M. Lauzon, J. R. Wu, G. Kennedy, K. Trybus, S. Lowey, C. Berger, "Myosin conformational states determined by single fluorophore polarization," Proc. Natl. Acad. Sci. USA 95, 8034-8039 (1998).

25. H. Sosa, E. J. G. Peterman, W. E. Moerner, L. S. B. Goldstein, "ADP-induced rocking of the kinesin motor domain revealed by single-molecule fluorescence polarization microscopy," Nat. Struct. Biol. 8, 540-544 (2001).

26. S. Abrahamsson, M. McQuilken, S. B. Mehta, A. Verma, J. Larsch, R. Ilic, R. Heintzmann, C. I. Bargmann, A. S. Gladfelter, R. Oldenbourg, "MultiFocus Polarization Microscope (MF-PolScope) for $3 \mathrm{D}$ polarization imaging of up to 25 focal planes simultaneously," Opt. Express 23, 7734-7754 (2015).

27. B. S. DeMay, X. Bai, L. Howard, P. Occhipinti, R. A. Meseroll, E. T. Spiliotis, R. Oldenbourg, A. S. Gladfelter, "Septin filaments exhibit a dynamic, paired organization that is conserved from yeast to mammals, Septin filaments exhibit a dynamic, paired organization that is conserved from yeast to mammals," J. Cell Biol. 193, 1065-1081 (2011).

28. B. S. DeMay, N. Noda, A. S. Gladfelter, R. Oldenbourg, "Rapid and quantitative imaging of excitation polarized fluorescence reveals ordered septin dynamics in live yeast," Biophys. J. 101, 985-994 (2011).

29. A. M. Vrabioiu, T. J. Mitchison, "Structural insights into yeast septin organization from polarized fluorescence microscopy," Nature 443, 466-469 (2006).

30. T. Ha, T. Enderle, S. Chemla, R. Selvin, S. Weiss, "Single molecule dynamics studied by polarization modulation," Phys. Rev. Lett. 77, 3979-3982 (1996).

31. G. Steinbach, I. Pomozi, D. P. Jánosa, J. Makovitzky, G. Garab, "Confocal fluorescence detected linear dichroism imaging of isolated human amyloid fibrils. Role of supercoiling," J. Fluoresc. 21, 983989 (2011).

32. X. Wang, A. Kress, S. Brasselet, P. Ferrand, "High frame-rate fluorescence confocal angle-resolved linear dichroism microscopy," Rev. Sci. Instrum. 84, 053708 (2013).

33. P. Ferrand, P. Gasecka, A. Kress, X. Wang, F. Z. Bioud, J. Duboisset, S. Brasselet, "Ultimate use of two-photon fluorescence microscopy to map orientational behavior of fluorophores," Biophys. J. 106, 2330-2339 (2014).
34. A. Gasecka, T. J. Han, C. Favard, B. R. Cho, S. Brasselet, "Quantitative imaging of molecular order in lipid membranes using two-photon fluorescence polarimetry," Biophys. J. 97, 2854-2862 (2009).

35. W. Li, Y. Wang, H. R. Shao, Y. H. He, H. Ma, "Probing rotation dynamics of biomolecules using polarization based fluorescence microscopy," Microsc. Res. Techniq. 70, 390-395 (2007).

36. J. R. Lakowicz, "Principles of fluorescence spectroscopy," J. Biomed. Opt. 13, 029901 (2008).

37. P. R. Callis, "The theory of two-photon-induced fluorescence anisotropy," In Topics in Fluorescence Spectroscopy, Vol. 5: Nonlinear and Two-PhotonInduced Fluorescence, J. R. Lakowicz, ed. (Boston, MA: Springer US), pp. 1-42 (2002).

38. S. Y. Chen, B. W. Van Der Meer, "Theory of twophoton induced fluorescence anisotropy decay in membranes," Biophys. J. 64, 1567-1575 (1993).

39. J. N. Forkey, M. E. Quinlan, Y. E. Goldman, "Protein structural dynamics by single-molecule fluorescence polarization," Prog. Biophys. Mol. Biol. 74, 1-35 (2000).

40. G. Weber, "Polarization of the fluorescence of macromolecules. I. Theory and experimental method," Biochem. J. 51, 145-155 (1952).

41. M. Ohmachi, Y. Komori, A. H. Iwane, F. Fujii, T. Jin, T. Yanagida, "Fluorescence microscopy for simultaneous observation of 3D orientation and movement and its application to quantum rod-tagged myosin V," Proc. Natl. Acad. Sci. U S A 109, 5294-5298 (2012).

42. J. Jasny, J. Sepiol, "Single molecules observed by immersion mirror objective. A novel method of finding the orientation of a radiating dipole," Chem. Phys. Lett. 273, 439-443 (1997).

43. J. Sepiol, J. Jasny, J. Keller, U. P. Wild, "ingle molecules observed by immersion mirror objective. The orientation of terrylene molecules via the direction of its transition dipole moment," Chem. Phys. Lett. 273, 444-448 (1997).

44. A. P. Bartko, R. M. Dickson, "Imaging threedimensional single molecule orientations," J. Phys. Chem. B 103, 11237-11241 (1999).

45. M. Bohmer, J. Enderlein, "Orientation imaging of single molecules by wide-field epifluorescence microscopy," J. Opt. Soc. Am. B 20, 554-559 (2003).

46. D. Patra, I. Gregor, J. Enderlein, "Image analysis of defocused single-molecule images for three-dimensional molecule orientation studies," J. Phys. Chem. A 108, 6836-6841 (2004).

47. Y. Sun, H. W. Schroeder, J. F. Beausang, K. Homma, M. Ikebe, Y. E. Goldman, "Myosin VI walks "wiggly" on actin with large and variable tilting," Mol. Cell 28, 954-964 (2007). 
48. M. P. Backlund, M. D. Lew, A. S. Backer, S. J. Sahl, G. Grover, A. Agrawal, R. Piestun, W. E. Moerner, "Simultaneous, accurate measurement of the 3D position and orientation of single molecules," Proc. Natl. Acad. Sci. USA 109, 19087-19092 (2012).

49. M. Tokunaga, N. Imamoto, K. Sakatasogawa, "Highly inclined thin illumination enables clear single-molecule imaging in cells," Nat. Methods 5, 159 (2008).

50. Y. Sun, O. Sato, F. Ruhnow, M. E. Arsenault, M. Ikebe, Y. E. Goldman, "Single-molecule stepping and structural dynamics of myosin X," Nat. Struct. Mol. Biol. 17, 485-491 (2010).

51. S. Inoué, O. Shimomura, M. Goda, M. Shribak, P. T. Tran, "Fluorescence polarization of green fluorescence protein," Proc. Natl. Acad. Sci. 99, 4272-4277 (2002).

52. N. Hafi, M. Grunwald, L. S. van den Heuvel, T. Aspelmeier, C. Steinem, M. Korte, A. Munk, P. J. Walla, "Reply to polarization modulation adds little additional information to super-resolution fluorescence microscopy," Nat. Methods 13, 8-9 (2016).

53. E. Abbe, "Beiträge zur theorie des mikroskopsageund der mikroskopischen Wahrnehmung," Arch. Mikrosk. Anat. 9, 413-418 (1873).

54. S. W. Hell, J. Wichmann, "Breaking the diffraction resolution limit by stimulated emission: Stimulatedemission-depletion fluorescence microscopy," Opt. Lett. 19, 780-782 (1994).

55. T. A. Klar, S. Jakobs, M. Dyba, A. Egner, S. W. Hell, "Fluorescence microscopy with diffraction resolution barrier broken by stimulated emission," Proc. Natl. Acad. Sci. USA 97, 8206-8210 (2000).

56. D. Dan, M. Lei, B. L. Yao, W. Wang, M. Winterhalder, A. Zumbusch, Y. J. Qi, L. Xia, S. H. Yan, Y. L. Yang, P. Gao, T. Ye, W. Zhao, "DMD-based LED-illumination Super-resolution and optical sectioning microscopy," Sci. Rep.-Uk 3 (2013).
57. M. G. Gustafsson, "Nonlinear structured-illumination microscopy: Wide-field fluorescence imaging with theoretically unlimited resolution," Proc. Natl. Acad. Sci. USA 102, 13081-13086 (2005).

58. M. G. L. Gustafsson, "Surpassing the lateral resolution limit by a factor of two using structured illumination microscopy," J. Microsc.-Oxford 198, 82-87 (2000).

59. E. Betzig, G. H. Patterson, R. Sougrat, O. W. Lindwasser, S. Olenych, J. S. Bonifacino, M. W. Davidson, J. Lippincott-Schwartz, H. F. Hess, "Imaging intracellular fluorescent proteins at nanometer resolution," Science 313, 1642-1645 (2006).

60. S. T. Hess, T. P. Girirajan, M. D. Mason, "Ultrahigh resolution imaging by fluorescence photoactivation localization microscopy," Biophys. J. 91, 4258-4272 (2006).

61. Z. L. Huang, H. Y. Zhu, F. Long, H. Q. Ma, L. S. Qin, Y. F. Liu, J. P. Ding, Z. H. Zhang, Q. M. Luo, S. Q. Zeng, "Localization-based super-resolution microscopy with an sCMOS camera," Opt. Express 19, 19156-19168 (2011).

62. M. J. Rust, M. Bates, X. W. Zhuang, "Subdiffraction-limit imaging by stochastic optical reconstruction microscopy (STORM)," Nat. Methods 3, 793-795 (2006).

63. L. Frahm, J. Keller, "Polarization modulation adds little additional information to super-resolution fluorescence microscopy," Nat. Methods 13, 7-8 (2016).

64. K. Ong, C. Wloka, S. Okada, T. Svitkina, E. Bi, Architecture and dynamic remodelling of the septin cytoskeleton during the cell cycle," Nat. Commun. 5, 5698 (2014).

65. R. Yasuda, H. Noji, M. Yoshida Jr., K. Kinosita, H. Itoh, "Resolution of distinct rotational substeps by submillisecond kinetic analysis of F1-ATPase," Nature 410, 898-904 (2001). 